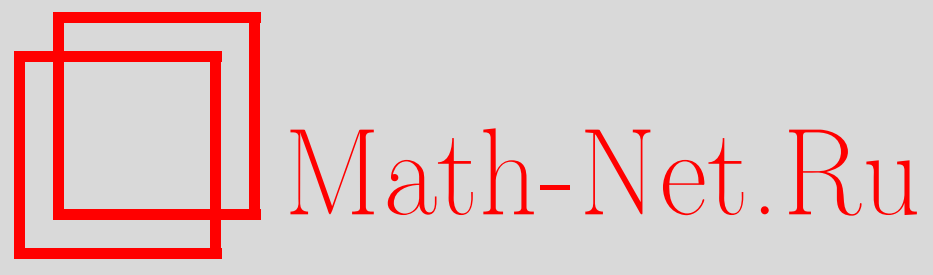

М. Г. Шур, Условие равномерной интегрируемости в сильных предельных теоремах для отношений. I, Теория вероятн. и ее примен., 2005, том 50, выпуск 3, 517-532

DOI: https://doi.org/10.4213/tvp92

Использование Общероссийского математического портала MathNet.Ru подразумевает, что вы прочитали и согласны с пользовательским соглашением

http://www.mathnet.ru/rus/agreement

Параметры загрузки:

IP : 54.157 .27 .8

26 апреля 2023 г., 14:13:52

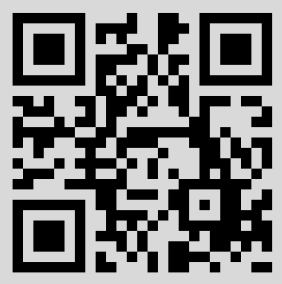




\section{УСЛОВИЕ РАВНОМЕРНОЙ ИНТЕГРИРУЕМОСТИ В СИЛЬНЫХ ПРЕДЕЛЬНЫХ ТЕОРЕМАХ ДЛЯ ОТНОШЕНИЙ. ${ }^{1)}$}

Задав цепь Маркова с измеримым пространством состояний $(E, \mathscr{E})$ и переходным оператором $P$ и зафиксировав измеримую функцию $f \geqslant 0$, мы при надлежаших условиях рассматриваем величины $\mu\left(f_{n}\right)$, где $n \geqslant 1$ достаточно велико, $f_{n}=P^{n} f / \nu\left(P^{n} f\right)$, а $\mu$ и $\nu$ являются вероятностными мерами на $\mathscr{E}$. Для широкого класса ситуаций предложены достаточные, а по большей части необходимые и достаточные условия сходимости $f_{n}$ к 1 при $n \rightarrow \infty$. Отличие этих результатов от принадлежащих Ори, Лину, Нуммелину и иным авторам проявляется в замене как традиционных условий типа возвратности цепи или равномерной ограниченности функций $f_{n}$, так и условия минорирования из [8] более гибкими предположениями, среди которых особую роль играет требование равномерной интегрируемости функций $f_{n}$ относительно некоторого набора мер. Наши теоремы влекут слабую, а зачастую и сильную сходимость этих функций к $\varphi \equiv 1$ в соответствующих пространствах суммируемых функций.

Ключевые слова и фразы: цепь Маркова, сильная предельная теорема для отношений.

\section{1. Введение}

1. На протяжении работы всюду, где нет особых оговорок, речь идет об (однородной) цепи Маркова $X=\left(X_{n} ; n \geqslant 0\right)$ с измеримым пространством состояний $(E, \mathscr{E})$ и переходным оператором $P$, действуюцим в пространстве $\mathscr{B}$ всех измеримых (т.е. $\mathscr{E}$-измеримых) ограниченных снизу функций $f: E \rightarrow(-\infty, \infty)$ (символ $\mathscr{B}_{b}$ будет обозначать семейство всех ограниченных $f \in \mathscr{B}$, а $\mathscr{B}_{+}-$семейство $\left.\left\{f \in \mathscr{B}_{b}: f \geqslant 0\right\}\right)$. Сильные предельные теоремы для отношений (СПТО) при надлежащих условиях доставляют информацию о поведении отношений $\mu\left(P^{n+m} f\right) / \nu\left(P^{n} g\right)$ с целым $m$ при $n \rightarrow \infty$, где $f, g \in \mathscr{B}_{+}$, меры $\mu$ и $\nu$ берутся из той или иной

* Московский государственный институт электроники и математики, Б. Трехсвятительский пер., 3/12, 109028 Москва, Россия.

1) Работа выполнена при поддержке РФФИ (грант № 04-01-169). 
части семейства $\mathscr{P}$ всех вероятностных мер на $\mathscr{E}$, а символ типа $\mu(\varphi)$ обозначает интеграл $\int \varphi d \mu$, коль скоро тот имеет смысл для какой-либо функции $\varphi$. Эта информация, содержащаяся, например, в приведенных ниже соотношениях (1.2) и (1.5), существенно уточняет утверждения таких классических предельных теорем для отношений, как предельная теорема Дёблина для счетных возвратных цепей Маркова (см. [10, § 1.9] или $[4$, гл. 4]) или эргодическая теорема Чакона-Орнстейна, сформулированная в терминах положительных сжатий пространств суммируемых функций (см. [7], [9]).

Первые СПТО связаны с именами К. Л. Чжуна, П. Эрдёша, С. Ори, Н. Джейна и других авторов. Среди соответствующих результатов, подытоженных в [4], наиболее отшлифованы, на наш взгляд, относящиеся к дискретным возвратным цепям Маркова, причем в качестве прекрасного введения в этот круг вопросов можно рекомендовать книгу [16], описывающую, в частности, связи между СПТО, проблемами суммирования независимых случайных величин и теорией потенциала. В последовавших СПТО чаще всего рассматривались цепи с измеримыми пространствами состояний, включая, в частности, возвратные по Харрису цепи, симметричные цепи, случайные блуждания на алгебраических структуpax (см., например, [2], [3], [8], 11]-[15]). Здесь мы напомним следующую СПТО из [8] (по поводу терминологии см. [8] или наш §4; пока лишь условимся целые неотрицательные числа обозначать латинскими буквами от $i$ до $n$ включительно и, работая с вещественными $a_{n}$ и $b_{n}$, $n \geqslant 0$, писать $a_{n} \sim b_{n}$, если $a_{n} / b_{n} \rightarrow 1$ при $\left.n \rightarrow \infty\right)$.

Теорема 1.1. Пусть $\sigma$-алгебра $\mathscr{E}$ счетнопорождена, а иепь $X$ возвратна по Харрису и апериодична. Пусть Функиии $\mu, \nu \in \mathscr{P} u$ мерь $f, g \in \mathscr{B}$ являются мальми и

$$
\nu\left(P^{n+1} f\right) \sim \nu\left(P^{n} f\right)
$$

Тогда при любом $m$

$$
\lim _{n \rightarrow \infty} \frac{\mu\left(P^{n+m} g\right)}{\nu\left(P^{n} f\right)}=\frac{\pi(g)}{\pi(f)},
$$

где $\pi$ - б-конечная инвариантная мера для $X(\pi(E)>0)$.

Отметим, что при осуществлении (1.2) для достаточно большого запаса функций $f, g$ и мер $\mu, \nu$ нередко говорят о наличии у $X$ свойства равнораспределенности относительно $\pi$.

При выводе соотношений типа (1.2) в ряде случаев удалось заменить требование возвратности по Харрису более слабыми (к примеру, условием Лиувилля; см. [2], [11]-[15]). Кроме того, как видно из указанных работ, проверка условия (1.1) - а в дискретном случае его ослабленного варианта [4] - часто вызывает затруднения. Поэтому представляет интерес следующая теорема из [13], являющаяся версией теоремы 
М. Лина [3] и отличающаяся от последней, например, отсутствием требований типа абсолютной непрерывности рассматриваемых мер.

Теорема 1.2. Пусть $f \in \mathscr{B}_{+} u \nu \in \mathscr{P}$ таковы, ито $\nu\left(P^{n} f\right)>0$ при всех $n$, начиная с некоторого натурального $n_{0}, u$

$$
\varliminf_{n \rightarrow \infty} \frac{\nu\left(P^{n+1} f\right)}{\nu\left(P^{n} f\right)} \geqslant 1
$$

Пусть семейство функиий $\mathscr{F}=\left\{f_{n} ; n \geqslant n_{0}\right\}$ равномерно ограничено в $E$, где $f_{n}=P^{n} f / \nu\left(P^{n} f\right), n \geqslant n_{0}, u$ nycmb

$$
\lim _{n \rightarrow \infty}\left\|(\mu-\nu) P^{n}\right\|=0
$$

для некоторой меры $\mu \in \mathscr{P}$. Тогда

$$
\mu\left(P^{n} f\right) \sim \nu\left(P^{n} f\right) .
$$

Если, помимо указанных условий, применимо и (1.4) с $\nu P$ вместо $\mu$, то

$$
\mu\left(P^{n+1} f\right) \sim \mu\left(P^{n} f\right) \quad u \quad \nu\left(P^{n+1} f\right) \sim \nu\left(P^{n} f\right) .
$$

Разумеется, справедливость (1.2) с $g=f$ и $g=P f$ автоматически влечет (1.5) и (1.6). Поэтому теоремы, обеспечивающие применимость соотношений вида (1.5) или (1.6), можно трактовать как простейшие СПТО. Они имеют самостоятельную ценность, и, как правило, из них достаточно просто выводятся обычные СПТО (см. [3], [13] и часть II данной работы). Что касается требования (1.4), при его реализации с любыми $\mu, \nu \in \mathscr{P}$ естественно говорить об осуществлении благоприятной возможности, предусмотренной вторым законом нуля-двойки в форме Дерриеника и гарантированной, в частности, для возвратных по Харрису цепей Маркова со счетнопорожденной $\sigma$-алгеброй $\mathscr{E}[9]$.

2. Основная задача данной части работы состоит в получении простейших СПТО с более гибкими условиями, нежели имеющиеся в приведенных теоремах. Так, равномерная ограниченность семейства $\mathscr{F}$ не гарантируется даже в довольно простых ситуациях (см. [3, теорема 2.4]), и поэтому в наших СПТО 2.1 и 2.2 используется требование равномерной интегрируемости $\mathscr{F}$ по некоторым мерам, а в СПТО 2.3 - его усиленный вариант. Кроме того, в отличие от [8], мы по возможности не предполагаем цепь $X$ ни возвратной по Харрису, ни удовлетворяющей условию минорирования из указанной книги, хотя при условиях из [8] рассматриваемый нами класс мер $\mathscr{M}_{1}(\nu)$ совпадает с классом малых мер в смысле [8]. Теоремы 2.1-2.4 мы считаем основными результатами работы (см. $\S 2$ и 3 ). Способы проверки не совсем привычных предположений этих теорем обсуждаются в $\S 4$, для чего пришлось внести некоторые уточнения в терминологию книги [8]. Читатель, готовый 
считать $\mathscr{E}$ счетнопорожденной и постулировать упоминавшееся условие минорирования, может игнорировать эти уточнения.

Во второй части работы мы обсудим, каким образом можно полностью избавиться от предположений типа (1.1).

Ввиду леммы 3.1 наши результаты, в основном, связаны со слабой предкомпактностью семейства $\mathscr{F}$ в некоторых пространствах суммируемых функций (хотя иногда слабую топологию удается заменить сильной; см. теорему 2.4). Поэтому мы активно используем известный критерий Данфорда-Петтиса слабой компактности [1], [5], устанавливающий связь между слабой предкомпактностью и равномерной интегрируемостью функциональных семейств.

\section{2. Обозначения и формулировки основных результатов}

1. Начнем с обозначений. В части, непосредственно касающейся основных результатов работы, используются терминология и обозначения, близкие к традиционным для теории цепей Маркова в измеримых пространствах состояний [4], [9], но позже потребуются некоторые уточнения (см. §4).

Как уже говорилось, в работе фиксируется измеримое пространство $(E, \mathscr{E})$. Под измеримыми функциями понимаются $\mathscr{E}$-измеримые функции на $E$, а под мерами - $\sigma$-конечные меры на $\mathscr{E}$, причем мера $\xi$ именуется тривиальной, если $\xi \equiv 0$. Совокупность всех мер обозначается $\mathscr{M}$.

Фиксируется также цепь Маркова $X=\left(X_{n} ; n \geqslant 0\right)$ с пространством состояний $(E, \mathscr{E})$ и одношаговыми переходными вероятностями $p(\cdot, \cdot) \equiv$ $p_{1}(\cdot, \cdot)$, и сохраняются уже введенные обозначения: $\mathscr{B}_{+}, \mathscr{P}, p_{n}(\cdot, \cdot)$ и т.д. Переходный оператор $P$ определим равенством $P f(x)=\int f(y) p(x, d y)$, $x \in E, f \in \mathscr{B}$, но иногда он будет истолковываться несколько иначе (см. $\S 3)$. Для $\alpha \geqslant 0$ и $f \in \mathscr{B}_{+}$положим

$$
G^{\alpha} f=\sum_{n \geqslant 0} \alpha^{n} P^{n} f
$$

и $G f=G^{1} f$. Для ограниченного заряда $\mu$ на $\mathscr{E}$ его норму $\|\mu\|$ приравняем к его вариации, т.е. к $\sup \{\mu(f) ; f \in \mathscr{B}$ и $|f| \leqslant 1\}$, а заряды $\mu P^{n}$, $n \geqslant 1$, определим равенством $\mu P^{n}(f)=\mu\left(P^{n} f\right), f \in \mathscr{B}_{+}$, распространив последнее определение и на любые меры $\mu$ на $\mathscr{E}$. Положим также $\mu G^{\alpha}(A)=\mu\left(G^{\alpha} 1_{A}\right)$, где $1_{A}$ - функция-индикатор измеримого множества $A$, а $\mu \in \mathscr{M}$ (разумеется, $\mu G^{\alpha} \notin \mathscr{M}$ при некоторых $\mu \in \mathscr{M}$ и $\alpha \geqslant 0$ ). Измеримая функция $f: E \rightarrow[0, \infty]$ называется гармонической (для $X$ ), если $f \equiv P f$ и $f \not \equiv \infty$. Задав нетривиальную меру $\xi \in \mathscr{M}$, назовем цепь $X$ обладающей $\xi$-свойством Лиувилля, коль скоро всякая гармоническая функция равна некоторой константе $\xi G^{\alpha}$-п.в. (т.е. $\xi G^{\alpha}$-поч- 
ти всюду) при каком-либо, а потому и всех $\alpha>0$. Всякая цепь Маркова, возвратная по Харрису, обладает $\xi$-свойством Лиувилля, если $\sigma$-алгебра $\mathscr{E}$ счетнопорождена и мера $\xi G^{\alpha}$ абсолютно непрерывна относительно соответствующей инвариантной меры [4], [9].

Выделим некоторые подсемейства семейства $\mathscr{M}$. Всякой мере $\nu \in \mathscr{M}$, для которой $\eta=\nu G^{\alpha} \in \mathscr{M}$ при некотором $\alpha \in(0,1)$, сопоставим семейство $\mathscr{M}(\nu)$ всех мер $\mu \ll \nu$ с ограниченными плотностями $d \mu / d \nu$ и семейство $\mathscr{M}_{1}(\nu)=\bigcup_{n \geqslant 0} \mathscr{M}\left(\nu P^{n}\right)$, а из этих семейств выделим соответствующие подсемейства вероятностных мер $\mathscr{P}(\nu)$ и $\mathscr{P} 1(\nu)$. Ясно, что если $\mu \in \mathscr{P}_{1}(\nu)$, то и $\mu P^{n} \in \mathscr{P}_{1}(\nu), n \geqslant 0$, и что $\mathscr{P}(\nu) \subset \mathscr{P}_{1}(\nu) \subset \mathscr{P}(\eta)$, благодаря чему, в частности $\mathscr{P}_{1}(\eta)=\mathscr{P}(\eta)$. Разумеется, любая мера $\mu \in \mathscr{P}(\eta)$ допускает плотность $h=d \mu / d \eta$ (этот факт мы отражаем в виде равенств $\mu=h \nu$ или $\mu=h \cdot \nu)$. Поэтому

$$
\mu=\sum_{m=0}^{\infty} \alpha^{m} \mu_{m}
$$

если $\mu$ имеет указанный вид, где $\mu_{m}=h \cdot \nu P^{m} \in \mathscr{M}_{1}(\nu)$.

2. Переходя к формулировкам основных результатов, назовем пару $\{f, \nu\}$, состоящую из каких-либо $f \in \mathscr{B}_{+}$и $\nu \in \mathscr{P}$, стандартной, если $\nu\left(P^{n} f\right)>0$ при всех $n$, начиная с некоторого $n=n_{0}$.

Далее в $\S \S 2$ и 3 фиксируется стандартная пара $\{f, \nu\}$ с соответствующим $n_{0}$, а под $\mathscr{F}$, как и в $\S 1$, подразумевается семейство $\left\{f_{n} ; n \geqslant n_{0}\right\}$, где $f_{n}=P^{n} f / \nu\left(P^{n} f\right)$. Задается и мера $\eta=\nu G^{\alpha}$ с $\alpha \in(0,1)$.

Мы предлагаем следующие необходимые и достаточные условия для реализации соотношения $\mu\left(P^{n} f\right) \sim \nu\left(P^{n} f\right)$, т.е. соотношения $(1.5)$ с подходящими $\mu$ (см. теоремы 2.1 и 2.2 , а также замечание 2.1 ).

Теорема 2.1. Пусть иепь $X$ обладает $\nu$-свойством Лиувилля (см. п.1). Тогда для справедливости (1.5) с произвольньлм $\mu \in \mathscr{P}(\eta)$ необходимо и достаточно, чтобь выполнялись условия:

(2A) семейство $\mathscr{F}$ равномерно интегрируемо по каждой мере $\nu P^{n}$, $n \geqslant 0$;

(2Б) $\partial л я$ всех $\mu \in \mathscr{P}_{1}(\nu)$

$$
\mu\left(P^{n+1} f\right) \sim \mu\left(P^{n} f\right)
$$

Из приведенных ниже условий $\left(2 \mathrm{Б}_{1}\right)$ и $\left(2 \mathrm{Б}_{2}\right)$ видно, что при проверке (2.3) довольно часто можно ограничиться случаем $\mu=\nu$, но иногда (см. $\left.\left(2 \mathrm{Б}_{1}\right)\right)$ можно избежать и этого. Простое условие, влекущее $(2 \mathrm{~A})$, указано в предложении 4.1.

3 а м е ч а н и е 2.1. а) Теорема останется в силе, если (1.5) усилить до соотношения $\mu\left(P^{n+m} f\right) \sim \nu\left(P^{n} f\right)$ с любыми $\mu \in \mathscr{P}(\eta)$ и $m \geqslant 1$. Действительно, в силу (1.5) и (2.3) $\mu\left(P^{n+1} f\right) \sim \nu\left(P^{n+1} f\right) \sim \nu\left(P^{n} f\right)$, 
если $\mu \in \mathscr{P}_{1}(\nu)$, а при рассмотрении прочих $\mu$ применимо следствие 3.1 (кстати, оно же позволяет в теореме заменить $\mathscr{P}(\eta)$ на $\mathscr{P}_{1}(\nu)$ ).

б) Как показано при доказательстве теоремы 2.1, она сохранит силу и тогда, когда условие 2Б заменяется условием

$\left(2 \mathrm{Б}_{1}\right)$ при $\mu=\nu$ применимо (2.3) (иньми словами, применимо (1.1)) u при любьлх $\mu \in \mathscr{P}_{1}(\eta)$

$$
\varlimsup_{n \rightarrow \infty} \frac{\mu\left(P^{n+1} f\right)}{\mu\left(P^{n} f\right)} \leqslant 1
$$

(при этом требование, касающееся (2.3), можно опустить, если иепь $X$ возвратна по Харрису, $\sigma$-алгебра $\mathscr{E}$ счетнопорождена $u \eta \ll \pi$, где $\pi$ - нетривиальная мера, инвариантная для $X)$.

Здесь вместо $\pi$ можно рассмотреть любую максимальную меру неприводимости цепи $X$ (см. $\S 4)$.

Первый пункт замечания 2.1 переносится и на теорему 2.2 .

Теорема 2.2. Теорема 2.1 останется в силе, если в ней вместо (2Б) использовать следуюшее требование:

$\left(2 \mathrm{Б}_{2}\right)$ при $\mu=\nu$ справедливо (2.1) (ср. с (2Б, $)$ ) и при всех $\mu \in \mathscr{P}_{1}(\nu)$

$$
\varliminf_{n \rightarrow \infty} \frac{\mu\left(P^{n+1} f\right)}{\mu\left(P^{n} f\right)} \geqslant 1 .
$$

Как показывает пример 1 из [13], следующая теорема является полезным дополнением теоремы 1.2 , причем доказательства обеих теорем сходны с доказательством теоремы 2.1 из [3].

Теорема 2.3. Пусть а) выполняется условие (2A) и найдется такое $M>0$, что $\nu P^{m}\left(A_{n}\right) \rightarrow 0, m \geqslant 0$, nрu $n \rightarrow \infty$, где $A_{n}=\{x \in E$ : $\left.f_{n}(x)>M\right\}, n \geqslant n_{0}$, б б) требование а) сохранит силу и при замене $\nu$ некоторой мерой $\mu \in \mathscr{P}$. Если вдобавок имеют место (1.3) и (1.4), то реализуется $u(1.5)$, а если еще выполняется (1.4) с $\nu P$ вместо $\mu$, то применимо и (1.6).

По лемме 3.1 осуществление (1.5) для всех $\mu \in \mathscr{P}_{1}(\nu)$ влечет для тех же и некоторых других $\mu$ слабую сходимость $f_{n}$ к 1 в $L(\mu)$ при $n \rightarrow \infty$. Нередко это заключение удается усилить следующим образом.

Tеорема 2.4. Пусть $\sup _{n \geqslant 1} q_{n}(x, E)=1$ для всех $x \in E$, где $q_{n}(x, \cdot)$ - абсолютно непрерьвная составляющая меры $p_{n}(x, \cdot)$ относительно $\eta$. Если (1.5) применимо с любьми $\mu \in \mathscr{P}_{1}(\nu)$, mо $\underline{\lim }_{n \rightarrow \infty} f_{n}=1$ всюду в $E$, причем $f_{n} \rightarrow 1$ в смьсле сильной сходимости в $L(\mu)$ для всех $\mu \in \mathscr{P}_{1}(\nu)$.

Условие, включенное в первую фразу этой теоремы, выполнено, в частности, если $q_{n}(x, E) \rightarrow 1$ всюду в $E$ при $n \rightarrow \infty$, что характерно, например, для многих случайных блужданий на группах и соответствующих $\nu$ (см. доказательство предложения 1.6 в [9, гл. 5]). 


\section{3. Доказательства основных результатов}

1. В этом параграфе мы продолжаем рассматривать некоторую стандартную пару $\{f, \nu\}$ вместе с соответствующими $n_{0}, f_{n}, \mathscr{F}$ и $\eta=\nu G^{\alpha}$, где $\alpha \in(0,1)$, причем начинаем с серии лемм.

Лемма 3.1. Если соотношение (1.5) справедливо для любых $\mu \in \mathscr{P}_{1}(\nu)$, то функиии $f_{n}$ слабо сходятся $\kappa 1$ при $n \rightarrow \infty$ в любом из пространств $L(\mu), \mu \in \mathscr{P}(\eta)$ (m.е. $\mu\left(f_{n} u\right) \rightarrow \mu(u)$ для $\left.u \in \mathscr{B}_{b}\right)$.

Д ок аз а т е л ь т в о. Пусть сначала $\xi \in \mathscr{P}$ и $\xi=g \cdot \nu P^{m}$ при некоторых $m \geqslant 0$ и $g \in \mathscr{B}_{+}$. Если $n \rightarrow \infty$, то

$$
\nu P^{m}\left(f_{n} g\right) \rightarrow \nu P^{m}(g),
$$

так как левая часть (3.1) при $n \geqslant n_{0}$ совпадает с величиной $\xi\left(f_{n}\right)$, стремящейся ввиду $(1.5)$ к 1 , а правая равна $\xi(E)=1$. Поэтому (3.1) применимо с любой функцией $g \in \mathscr{B}_{+}$, для которой $P^{m}(g)>0$, и то же можно сказать, если $g=0 \nu P^{m}$-п.в. Теперь из (3.1) следует утверждение леммы для $\mu=\nu P^{m}, m \geqslant 0$, а вместе с тем и для всех $\mu \in \mathscr{M}_{1}(\nu)$.

Пусть известно лишь, что $\mu \in \mathscr{P}(\eta)$, и пусть $\alpha(1+\varepsilon)<1$, где $\varepsilon>0$. В соответствии с $(1.5)$ отношения $\nu\left(P^{m+1} f_{n} / \nu\left(P^{m} f_{n}\right)=\right.$ $\nu\left(P^{m+n+1} f\right) / \nu\left(P^{m+n} f\right)$ и $\nu\left(P^{N+n} f\right) / \nu\left(P^{n} f\right)=\nu\left(P^{N} f_{n}\right)$ стремятся к 1 при $m+n \rightarrow \infty$ и $n \rightarrow \infty$ соответственно, где $N$ - натуральное число. Поэтому при некотором $N_{1}>0$ и всех $N>N_{1}$ они не превосходят $1+\varepsilon$, если $n$ превышает некое $n_{1}=n_{1}(N) \geqslant n_{0}$ (вскоре выбор $N_{1}$ будет у'гочнен), и при $m>N>N_{1}$ и $n \geqslant n_{1}$ мы приходим к неравенству $\nu\left(P^{m} f_{n}\right) \leqslant(1+\varepsilon)^{m-N} \nu\left(P^{N} f_{n}\right)$, согласно которому

$s_{n} \equiv \sum_{m \geqslant N+1} \alpha^{m} \mu_{m}\left(f_{n}\right) \leqslant a \sum_{m \geqslant N+1} \alpha^{m} \nu\left(P^{m} f_{n}\right) \leqslant b \alpha^{N} \nu\left(P^{N} f_{n}\right) \leqslant(1+\varepsilon) b \alpha^{N}$, где $\mu_{m}=h \cdot \nu P^{m}$ - те же, что и в $(2.2), a=\sup h, b=a[1-\alpha(1+\varepsilon)]^{-1}$ и $n \geqslant n_{1}$. Отсюда вывод: $s_{n}<\varepsilon$ при $n \geqslant n_{1}$ и $N \geqslant N_{1}$, если $N_{1}$ выбрано так, чтобы $(1+\varepsilon) b \alpha^{N_{1}}<\varepsilon$, что мы и предположим впредь.

Пусть $u \in B_{+}$и $|u| \leqslant 1$. Наш вывод ввиду (2.2) влечет неравенства

$$
\left|\mu\left(u f_{n}\right)-\sum_{0 \leqslant m \leqslant N} \alpha^{m} \mu_{m}\left(u f_{n}\right)\right| \leqslant s_{n}<\varepsilon \quad\left(N \geqslant N_{1}, n \geqslant n_{1}\right) .
$$

Но $\mu_{m} \in \mathscr{M}_{1}(\nu)$ и, следовательно, $\mu_{m}\left(u f_{n}\right) \rightarrow \mu_{m}(u)$ при $n \rightarrow \infty$ (см. начало доказательства). По этой причине $\mu\left(u f_{n}\right)$ отличается от $\sigma_{N}=\sum_{0 \leqslant m \leqslant N} \alpha^{m} \mu_{m}(u)$ не больше, чем на $2 \varepsilon$, при заданном $N \geqslant N_{1}$ и достаточно большом $n$. Увеличив же при необходимости $N$, можем с учетом (2.2) считать, что $\left|\mu(u)-\sigma_{N}\right|<\varepsilon$. Таким образом, $\mu\left(u f_{n}\right) \rightarrow \mu(u)$ при $n \rightarrow \infty$, и лемма доказана.

Следствие 3.1. Если соотношение (1.5) выполняется для любых $\mu \in \mathscr{P}_{1}(\nu)$, то оно справедливо и для всех $\mu \in \mathscr{P}(\eta)$. 
2. Всюду в этом пункте постулируется условие (2A). Кроме того, принимаются следующие соглашения. Символами $\mathfrak{N}, \mathfrak{N}_{1}, \mathfrak{M}$ и т.п. будут обозначаться возрастающие последовательности натуральных чисел, каждое из которых не меньше введенного выше $n_{0}$. Запись типа $\ll g_{n} \stackrel{\mathrm{w}}{\rightarrow} g$ в $L(\mu), n \in \mathfrak{N} \gg$, где $\mu$ - некоторая конечная мера, будет подразумевать, что $g_{n}, g \in L(\mu), n \in \mathfrak{N}$ и функции $g_{n}$ слабо сходятся в $L(\mu)$ к $g$, когда $n \rightarrow \infty$, пробегая $\mathfrak{N}$.

Лемма 3.2. Каждая последовательность $\left\{f_{n} ; n \in \mathfrak{N}\right\}$ содержит подпоследовательность $\left\{f_{n} ; n \in \mathfrak{M}\right\}$, слабо сходяшуюся в любом из пространств $L(\mu), \mu \in \mathscr{P}_{1}(\nu), \kappa$ некоторой функиии $F$, одной и той же для указанных $\mu$.

Д ок азательст в о. Сначала, задав $m \geqslant 0$, допустим, что $f_{n} \stackrel{\text { w }}{\rightarrow} F$ в $L\left(\nu_{m}\right), n \in \mathfrak{M}$, при подходящем выборе $F$ и $\mathfrak{M}$, где $\nu_{m}=$ $\sum_{0 \leqslant i \leqslant m} \nu P^{i}$. Тогда $\nu P^{i}=h_{i} \nu_{m}$ с некоторыми $h_{i} \in \mathscr{B}_{+}, 0 \leqslant i \leqslant m$, и для $u \in \mathscr{B}_{b}$ получаем

$$
\nu P^{i}\left(u f_{n}\right)=\nu_{m}\left(u h_{i} f_{n}\right) \longrightarrow \nu_{m}\left(u h_{i} F\right)=\nu P^{i}(u F),
$$

когда $n \rightarrow \infty$ вдоль $\mathfrak{M}$. Иными словами, в нашем случае $f_{n} \stackrel{\text { w }}{\rightarrow} F$ в $L\left(\nu P^{i}\right), n \in \mathfrak{M}$, при $0 \leqslant i \leqslant m$, чем вскоре мы и воспользуемся.

Опираясь на упоминавшийся в $\S 1$ критерий Данфорда-Петтиса, выделим из $\mathfrak{N}$ одну за другой подпоследовательности $\mathfrak{N}_{0} \supset \mathfrak{N}_{1} \supset \cdots$, для которых $f_{n} \stackrel{\text { w }}{\rightarrow} F_{m}$ в $L\left(\nu_{m}\right), n \in \mathfrak{N}_{m}$, при подходящем выборе функций $F_{m} \geqslant 0, m \geqslant 0$. Так как меры $\nu_{i}, 0 \leqslant i \leqslant m$, допускают плотности $h_{i m}=d \nu_{i} / d \nu_{m} \leqslant 1$, то $\nu_{i}\left(u f_{n}\right)=\nu_{m}\left(u h_{i m} f_{n}\right)$ для $u \in \mathscr{B}_{b}$. Отсюда следует (cp. $(3.2))$, что $\nu_{i}\left(u F_{i}\right)=\nu_{m}\left(u h_{i m} F_{m}\right)=\nu_{i}\left(u F_{m}\right)$ для тех же $u$, а это возможно лишь при $F_{i}=F_{m} \nu_{i}$-п.в., $i \geqslant 0$. Итак, $f_{n} \stackrel{\text { w }}{\longrightarrow} F$ в $L\left(\nu_{m}\right), n \in \mathfrak{N}_{m}$ при всех $m \geqslant 0$, где, например, можно взять $F=\underline{\lim }_{m \rightarrow \infty} F_{m}$. В этой фразе допустимо $\mathfrak{N}_{m}$ заменить «диагональной» последовательностью $\mathfrak{M}$, образующейся при выписывании один за другим первого элемента из $\mathfrak{N}_{0}$, второго из $\mathfrak{N}_{1}$ и т.д., после чего, вспомнив начало доказательства, получаем утверждение леммы для любых $\mu=\nu P^{m}, m \geqslant 0$, что и требовалось.

Чтобы подготовиться к следующему доказательству, рассмотрим меру $\xi$, удовлетворяющую условию:

$$
\xi P \leqslant C \xi
$$

при некотором $C>0$ (в число таких мер входят, например, инвариантные меры, для которых $C=1$, и меры $\xi=\mu G^{\alpha}$ с $\mu \in \mathscr{P}$, для которых можно взять $\left.C=\alpha^{-1}\right)$. Это условие позволяет трактовать $P$ как ограниченный оператор, действующий в пространстве $L(\mu)$ (ср. [3], [7], [9]). Точнее, если $\hat{f}$ - класс функций, эквивалентных (относительно $\xi$ ) функции $f$, суммируемой по $\xi$, то $P f$ определяют как класс функций, экви- 
валентных функции $\int f(y) p(\cdot, d y)$, заведомо определенной $\xi$-п.в. Тем самым, сохранив обозначение $P$, получают новый линейный оператор $P$, действующий в $L(\xi)$, с нормой $\|P\| \leqslant C$. Ему сопоставляется двойственный оператор $P^{*}$, действующий в $L_{\infty}(\xi)$, с нормой $\left\|P^{*}\right\|=\|P\|$, для которого $\xi\left(f P^{*} g\right)=\xi(g P f)$, если $f \in L(\xi)$ и $g \in L_{\infty}(\xi)[5],[7],[9]$.

При этом стоит отметить, что если некоторая мера $\nu \in \mathscr{M}$ имеет ограниченную плотность $g=d \nu / d \xi$, то $\xi$-п.в.

$$
P^{* n} g=\frac{d\left(\nu P^{n}\right)}{d \xi}
$$

при $n \geqslant 0$ (разумеется, $\left.P^{* n}=\left(P^{*}\right)^{n}\right)$. В самом деле, заставив $f$ пробегать $\mathscr{B}_{+}$, выводим (3.4) из равенств

$$
\nu\left(P^{n} f\right)=\xi\left(g P^{n} f\right)=\xi\left(f P^{* n} g\right) .
$$

Лемма 3.3. Если $\mathfrak{N}, \mathfrak{M}, F$ и $е$ те же, что и в предыдущей лемме, mo $P^{m} f_{n} \stackrel{\mathrm{w}}{\rightarrow} P^{m} F$ в $L(\mu), n \in \mathfrak{M}$, дде $m \geqslant 0$.

Д ок а з а т ел ь с в о. Сначала заметим, что

$$
\nu P^{i}\left(u P^{m} \varphi\right)=\nu P^{m+i}(v \varphi)
$$

если $i, m \geqslant 0, u, \varphi \in \mathscr{B}_{b}$, а $v$ приравнено к отношению $P^{* m}\left(u P^{* i} g\right) / P^{*(m+i)} g$ там, где его знаменатель отличен от 0 , и к 0 в остальных точках (здесь $P^{*}$ - только что введенный оператор, отвечающий мере $\xi=\eta$, а $g=d \nu / d \eta)$. Действительно, согласно (3.4) или (3.5), левая часть (3.6) совпадает с

$$
\eta\left(\left(u P^{m} \varphi\right) P^{* i} g\right)=\eta\left(\varphi P^{* m}\left(u P^{* i} g\right)\right)=\eta\left(\varphi v P^{*(m+i)} g\right)
$$

что опять же в силу (3.4) равно правой части (3.6).

Далее, по условию леммы $f_{n} \stackrel{\mathrm{w}}{\rightarrow} F$ в $L\left(\nu P^{m+i}\right), n \in \mathfrak{M}$, при $i, m \geqslant 0$, и, следовательно, $P^{m} F \in L\left(\nu P^{i}\right)$ и величина $\nu P^{m+i}\left(v f_{n}\right)=\nu P^{i}\left(u P^{m} f_{n}\right)$ стремится к $\nu P^{m+i}(v F)=\nu P^{i}\left(u P^{m} F\right)$, если $u \in \mathscr{B}_{b}$ и $n \rightarrow \infty$ вдоль $\mathfrak{M}$, где оба указанные равенства вытекают из (3.6). Это сразу влечет утверждение леммы для $\mu=\nu P^{i}, i \geqslant 0$, а затем и для остальных $\mu \in \mathscr{P}_{1}(\nu)$.

Д ок аз а т ел ь с т в о т е о р е м ы 2.1. Если соотношение (1.5) справедливо для любых $\mu \in \mathscr{P}_{1}(\nu)$, то очевидным образом выполняется и условие (2Б), тогда как (2А) вытекает из леммы 3.1 и критерия Данфорда-Петтиса. Иными словами, условия (2А) и (2Б), а потому $\left(2 \mathrm{Б}_{1}\right)$ и $\left(2 \mathrm{Б}_{2}\right)$ необходимы для выполнения $(1.5)$ с любыми $\mu \in \mathscr{P}_{1}(\nu)$.

Постулируем условия $(2 \mathrm{~A})$ и $\left(2 \mathrm{Б}_{1}\right)$ и установим их достаточность (тем самым будет обоснован и п. б) замечания 2.1). Если из $\mathscr{F}$ извлечена какая-либо последовательность $\left\{f_{n} ; n \in \mathfrak{N}\right\}$, то по лемме $3.3 \mathfrak{N}$ содержит 
такую подпоследовательность $\mathfrak{M}$, что $P^{m} f_{n} \stackrel{\text { w }}{\rightarrow} P^{m} F$ в $L(\mu), n \in \mathfrak{M}$, где $F \geqslant 0$ - некоторая измеримая функция, $m \geqslant 0$ и $\mu$ пробегает $\mathscr{P}_{1}(\nu)$. Поэтому нам достаточно установить, что

$$
F=1 \quad \mu \text {-п.в. }
$$

для указанных $\mu$ независимо от конкретного выбора $\mathfrak{N}$ и $\mathfrak{M}$ рассматриваемого типа.

С этой целью, задав $\mathfrak{N}, \mathfrak{M}$ и $\mu$ с требуемыми свойствами, докажем, что

$$
P^{m+1} F \leqslant P^{m} F \quad \mu \text {-п.в. }
$$

при $m \geqslant 0$. В самом деле, если $\xi=u \mu, u \in \mathscr{B}_{+}$и мера $\xi$ нетривиальна, то (2.4) применительно к $\xi$ показывает, что

$$
\mu\left(u P^{m+1} f_{n}\right)=\xi\left(P^{m+1} f_{n}\right) \leqslant(1+\varepsilon) \xi\left(P^{m} f_{n}\right)=(1+\varepsilon) \mu\left(u P^{m} f_{n}\right)
$$

при заданных $i, m \geqslant 0, \varepsilon>0$ и достаточно больших $n$. Отсюда вытекает неравенство $\mu\left(u P^{m+1} F\right) \leqslant(1+\varepsilon) \mu\left(u P^{m} F\right)$, а вслед за ним и (3.8), так как выбор $\varepsilon$ и $u$ допускает достаточную свободу. (Для последующего отметим, что если вместо $\left(2 \mathrm{Б}_{1}\right)$ ввести $\left(2 \mathrm{Б}_{2}\right)$, то сходным образом можно получить (3.8), но со знаком неравенства, обращенным в другую сторону.)

Рассмотрим $\mu \in \mathscr{P}_{1}(\nu)$ и множества $A=\left\{x \in E: P^{m+1} F(x) \leqslant\right.$ $\left.P^{m} F(x), m=0,1,2, \ldots\right\}, B=E \backslash A$ и $D=\left\{x \in A: G^{\alpha}(x, B)=0\right\}$. В соответствии с (3.8) $\mu P^{n}(B)=0, n \geqslant 0$, так как $\mu P^{n} \in \mathscr{P}_{1}(\nu)$. Таким образом, $\mu G^{\alpha}(B)=0$, а отсюда следует, что $\mu(A \backslash D)=0$ и

$$
\mu(E \backslash D)=\mu(B \cup(A \backslash D))=0 .
$$

Множество $D$ является поглощающим в том смысле, что на нем $p(\cdot, D) \equiv 1$ (см. [8, предложение 2.2$])$, благодаря чему функция $F_{0}$, совпадающая с $F$ на $D$ и с $+\infty$ вне $D$, оказывается супергармонической (т.е. $0 \leqslant P F_{0} \leqslant F_{0}$ и $\left.F_{0} \not \equiv \infty\right)$. Равенство $F=F_{0} \mu$-п.в., $\mu \in \mathscr{P}_{1}(\nu)$, вытекающее из (3.9), и определение слабой сходимости дают право далее считать саму функцию $F$ супергармонической. Если реализуются условия, указанные в $\left(2 \mathrm{Б}_{1}\right)$ после $(2.4)$, то из свойств функций, супергармонических для возвратных по Харрису цепей Маркова (см. [9, гл. 3]), вытекает равенство $F=d \pi$-п.в., а за ним и равенство $F=d \eta$-п.в., где $d \geqslant 0$ постоянно, а $\pi$ - соответствующая инвариантная мера. Сопоставив очевидные соотношения $\nu\left(f_{n}\right) \rightarrow \nu(F)=d$, где $n \rightarrow \infty$ вдоль $\mathfrak{M}$, и $\nu\left(f_{n}\right)=1$, где $n \geqslant n_{0}$, приходим к равенству $F=1 \eta$-п.в., влекущему (3.7).

Теперь откажемся от только что использованной части требований из $\left(2 \mathrm{Б}_{1}\right)$. Если $n \rightarrow \infty$, пробегая $\mathfrak{M}$, то по лемме $3.3 \nu P^{m}\left(f_{n}\right) \rightarrow \nu\left(P^{m} F\right)$, 
а по условию $\left(2 \mathrm{Б}_{1}\right) \nu P^{m+1}\left(f_{n}\right) / \nu P^{m}\left(f_{n}\right)=\nu P^{m+n+1}(f) / \nu P^{m+n}(f) \rightarrow 1$, и, таким образом, $\nu P^{m+1}(F)=\nu P^{m}(F), m \geqslant 0$. Так как $F$ супергармонична, функции $P^{n} F$, не возрастая с ростом $n$, сходятся к некоторой функции $\widetilde{F}$, причем $0 \leqslant \widetilde{F} \leqslant F$. Полученное только что равенство и теорема Лебега о переходе к пределу под знаком интеграла показывают при $m \geqslant 0$, что, во-первых,

$$
\nu P^{m}(\widetilde{F})=\lim _{n \rightarrow \infty} \nu P^{m+n}(F)=\nu P^{m}(F)
$$

и, стало быть, $\eta(F-\tilde{F})=0$ т.е. $\widetilde{F}=F \eta$-п.в., и, во-вторых, $\tilde{F}=P^{m} \tilde{F}$ на поглощающем множестве $D_{1}=\{x \in E: F(x)<\infty\}$, для которого $\eta\left(E \backslash D_{1}\right)=0$. Далее, положив $\varphi_{1}=\widetilde{F}$ на $D_{1}$ и $\varphi_{1}=0$ вне $D_{1}$ и отметив неравенства $F \geqslant \varphi_{1}$ и $P \varphi_{1} \geqslant \varphi_{1}$, заключаем, что $F \geqslant P^{n} F \geqslant$ $P^{n} \varphi_{1} \geqslant P^{n-1} \varphi_{1}, n \geqslant 1$. Следовательно, функции $P^{n} \varphi_{1}$ при $n \rightarrow \infty$ снизу сходятся к некоторой функции $\varphi \leqslant F$, которая гармонична для $X$, что легко усганавливается с помощью теоремы Б. Леви о предельном переходе, и совпадает с $\varphi_{1}=\widetilde{F}$ на $D_{1}$, а вместе с тем и с $F \eta$-п.в. Из свойства Лиувилля цепи $X$ следует равенство $\varphi=d \eta$-п.в., где снова $d \geqslant 0$ постоянно. Таким образом, $F=d \eta$-п.в., а отсюда, как это уже делалось, выводится (3.7). Теорема доказана.

Д о к аз а т ел с с в о т е о ре мы 2.2. Необходимость условий $(2 \mathrm{~A})$ и $\left(2 \mathrm{Б}_{2}\right)$ уже установлена в начале предыдущего доказательства, и здесь мы, предположив эти условия выполненными, докажем (1.5).

Задача вновь сводится к проверке (3.7) для прежних $\mathfrak{N}, \mathfrak{M}, F$ и $\mu$. Как отмечено при выводе (3.8), вместо (3.8) теперь применимо неравенство $P^{m+1} F \geqslant P^{m} F \mu$-п.в. для $\mu \in \mathscr{P}_{1}(\nu)$, и, следовательно, функции $P^{n} F$ на множестве $A_{1}=\left\{x \in E: P^{m+1} F(x) \geqslant P^{m} F(x) ; m \geqslant 0\right\}$ при $n \rightarrow \infty$ сходятся снизу к некоторой функции $\widetilde{F}: A_{1} \rightarrow[0, \infty]$, причем $\mu\left(E \backslash A_{1}\right)=0$ для тех же $\mu$. Приравняв $\widetilde{F}$ к 0 на $E \backslash A_{1}$ и повторив с небольшими изменениями вывод (3.10), получаем равенства $\nu P^{m}(\widetilde{F})=\nu P^{m}(F), m \geqslant 0$, ввиду которых $\widetilde{F}=F \eta$-п.в. (упомянутые изменения вызваны лишь тем, что теперешняя функция временно не может считаться супергармонической и что вместо теоремы Лебега здесь удобнее привлечь теорему Б. Леви). На основании сказанного $P^{m+1} F=P^{m} F$, $m \geqslant 0, \mu$-п.в., если $\mu \in \mathscr{P}_{1}(\nu)$. Таким образом, установлен усиленный вариант (3.8), позволяющий вновь вывести (3.7), использовав (2.3) с $\nu$ в роли $\mu$ и немного упростив конец предыдущего доказательства.

Д оказательств о те о ремы 2.3. Временно исключим условие теоремы, касающееся $\nu P$. Если $m \geqslant 1$ задано, а $n$ достаточно велико, то для $J_{n}=\left|\mu\left(P^{n} f\right)-\nu\left(P^{n} f\right)\right|$ на основании (1.3) находим:

$$
\begin{aligned}
J_{n} & =\left|(\mu-\nu) P^{m} P^{n-m}(f)\right| \leqslant\left(I_{1}+I_{2}\right) \nu\left(P^{n-m} f\right) \\
& \leqslant 2\left(I_{1}+I_{2}\right) \nu\left(P^{n} f\right),
\end{aligned}
$$


где $I_{1}=\left|(\mu-\nu) P^{m}\left(f_{n-m} 1_{A_{n-m}}\right)\right|$, а $I_{2}$ определено аналогично, но с множеством $A_{n-m}$, замененным его дополнением (множества $A_{n}$ и соответствующее $M>0$ определены в тексте теоремы). Из предположений а) и б) следует, что $I_{1} \rightarrow 0$ при $n \rightarrow \infty$ и любом $m \geqslant 0$. Величину же $I_{2}$ можно сделать сколь угодно близкой к 0 , подобрав $m$ с учетом (1.4) и неравенства $I_{2} \leqslant M\left\|(\mu-\nu) P^{m}\right\|$. Поэтому из $(3.11)$ вытекает, что $J_{n} / \nu\left(P_{n} f\right) \rightarrow 0$ при $n \rightarrow \infty$, откуда и выводится (1.5).

Если теперь ввести исключенное вначале условие, то получим, в частности, (1.5) с $\nu P$ вместо $\mu$. Тем самым будет обосновано второе, а с ним в силу (1.5) и первое из соотношений (1.6).

Доказательство теоремы 2.4. Зададим $x \in E$ и $\varepsilon \in\left(0, \frac{1}{2}\right)$. Положив $\widetilde{q}_{n}(y)=q_{n}(x, d y) / \eta(d y), y \in E, n \geqslant 1$, подберем $m \geqslant 1$, а затем $C \geqslant 1$ из условий $q_{m}(x, E)>1-\varepsilon$ и $\eta(g)>1-2 \varepsilon$ соответственно, где $g(\cdot)=\min \left(\widetilde{q}_{m}(\cdot), C\right)$. Ввиду $(1.5)$ при достаточно больших $n$

$f_{n}(x)=\frac{\nu\left(P^{n-m} f\right)}{\nu\left(P^{n} f\right)} P^{m} f_{n-m}(x) \geqslant(1-\varepsilon) \eta\left(g f_{n-m}\right) \geqslant(1-\varepsilon)^{2} \eta(g)>(1-2 \varepsilon)^{3}$,

поскольку функции $f_{n}$ слабо сходятся к 1 в $L(\eta)$ (см. лемму 3.1 ). Таким образом, $\underline{\lim }_{n \rightarrow \infty} f_{n}=1$ всюду в $E$, что в сочетании с упомянутой слабой сходимостью функций $f_{n}$ влечет их сильную сходимость к 1 (см. $[1$, теорема II.26]).

\section{4. Дополнительные результаты}

В связи с основными результатами работы (см. § 2) встает вопрос о способах проверки включенных в них требований, и мы обсуждаем его в последующих пп. 2 и 3 , но сначала в п. 1 остановимся на терминологии, связанной с неприводимыми цепями Маркова.

1. Понятие неприводимой (точнее, неприводимой с мерой неприводимости $\xi$ ) цепи Маркова считается известным [4], [8]. Для такой цепи в качестве $\xi$ всегда можно взять любой элемент множества $\mathscr{M}_{0}$ всех максимальных мер неприводимости, т.е. меру неприводимости $\xi$ всегда можно выбрать так, чтобы $P G 1_{A}=0 \xi$-п.в., если $A \in \mathscr{E}$ и $\xi(A)=0$. При этом все меры из $\mathscr{M}_{0}$ эквивалентны друг другу (см. [8, предложение 2.7]).

Аналоги приводимых далее определений привязаны в [8] к ситуации, когда цепь $X$ неприводима, $\sigma$-алгебра $\mathscr{E}$ счетнопорождена и реализуется условие минорирования, упомянутое в п. 2 введения, что заставляет нас несколько обобщить соответствующие понятия из [8].

Пусть цепь $X$ неприводима и $\xi \in \mathscr{M}_{0}$. Эта цепь, по определению, является апериодической, если, каковы бы ни были $f \in \mathscr{B}_{+}$с $\xi(f)>0$ и $x \in E$, неравенство $P^{n} f(x)>0$ осушествляется при всех $x \in E$, начиная с некоторого $n_{1}$, которое может зависеть от выбора как $f$, так 
и $x$. В случае счетнопорожденной $\sigma$-алгебры $\mathscr{E}$ последнее определение эквивалентно принятому в [4].

Функция $f \in \mathscr{B}_{+}$называется малой, если $\xi(f)>0$ и для любой функции $g \in \mathscr{B}_{+}$с $\xi(g)>0$ найдутся такие $a>0$ и целое $m \geqslant 0$, что $f \leqslant a P^{m} g$. Это определение имеет много общего с определением очень малой функции [3]; в отечественной литературе малые функции называют также минорантными.

Пусть для $X$ найдется хотя бы одна малая функция (так обстоит дело, например, в недавно описанной ситуации из [8]). Тогда (см. [13]) существует параметр сходимости $R$ цепи $X$, т.е. постоянная $R \in[1, \infty)$ со свойствами: а) $G^{\alpha} f<\infty$ на некотором поглощающем множестве, если функция $f$ мала и $\alpha \in[0, R)$; б) $G^{\alpha} f \equiv \infty$ в $E$, если $\alpha>R, f \in \mathscr{B}_{+}$и $\xi(f)>0$.

2. Следующие утверждения предназначены для облегчения проверки требований типа (1.3), (2А) и (2Б) (теорема 4.1 является следствием теоремы 5 из [13] и ее доказательство опущено; остальные доказательства см. в п. 3).

Теорема 4.1. Пусть цепь Маркова $X$ явллется неприводимой $u$ апериодической и имеет параметр сходимости $R=1$. Пусть, кроме того, ее переходные вероятности подчинены следующему условию минорирования: существуют натуральное $N$ и ядра $q_{n}(x, A) \geqslant 0$, где $x \in E, A \in \mathscr{E}, n \geqslant N$, maкuе, чmо $\inf _{E} q_{n}(\cdot, E)>0$ u $p_{m}(\cdot, \cdot) \geqslant q_{n}(\cdot, \cdot)$ при всех $n \geqslant N$ и $m \in[N, n]$. Если для $X$ найдется хотя бы одна малая функиия, то (1.3) выполняется всякий раз, когда $\nu \in \mathscr{P}, f \in \mathscr{B}_{+} u$ $\xi(G f)>0$, әде $\xi \in \mathscr{M}_{0}$.

Ясно, что эта теорема может оказаться полезной и при проверке условия $\left(2 \mathrm{~b}_{2}\right)$. Упомянутое в ней условие минорирования выполнено, например, для многих случайных блужданий на группах [9], [13] и для дискретных апериодических цепей Маркова, удовлетворяющих условию Кингмана-Ори из принадлежещей им СПТО [4].

Предложение 4.1. Пусть мера $\xi$ удовлетворяет условию (3.3). Пусть $\nu \in \mathscr{P}(\xi)$ и сушествуют множества $E_{n} \in \mathscr{E}, n \geqslant 1$, со свойствами: а) $E_{1} \subset E_{2} \subset \cdots, E=\bigcup_{n \geqslant 1} E_{n} u \xi\left(E_{n}\right)<\infty ;$ б) любая из мер $\nu P^{n}, n \geqslant 0$, сосредоточена на некотором $E_{i}$, аде $i=i(n)$. Eсли семейство $\mathscr{F}$ (см. §2) равномерно интегрируемо по $\xi$ на каждом $E_{n}$, то реализуется (2A).

3 а м е ч а н и е 4.1. Рассмотрим апериодическую цепь Маркова со счетным множеством состояний, сообщающихся друг с другом (в качестве $\mathscr{E}$ берется совокупность всех подмножеств множества $E$ ). Предположив, что функция $f \geqslant 0$ и меры $\nu \in \mathscr{P}$ и $p(x, \cdot), x \in E$, имеют конечные носители, и воспользовавшись уравнением КолмогороваЧепмена, легко вывести (2А), коль скоро $f \not \equiv 0$ и последовательность 
$\left\{p_{n+1}(x, x) / p_{n}(x, x) ; n \geqslant N\right\}$ ограничена при каком-либо $x \in E$, где $p_{n}(x, x)=p_{n}(x,\{x\})$, а $N>0$ таково, что $p_{n}(x, x)>0$ при $n \geqslant N$ (примеры подобных неограниченных последовательностей см. в [4] и [10]).

Договоримся причислять цепь $X$ к классу $\mathscr{X}$, если найдутся $a>0$ и натуральные $m>l$, при которых $p_{m}(\cdot, \cdot) \geqslant a p_{l}(\cdot, \cdot)$, и в этом случае положим $q=m-l$ и $Q=P^{q}$. Счетные цепи введенного класса впервые появились в [6]; более общий случай см. в [2] и [11].

Предложение 4.2. Пусть иепь $X$ а) неприводима, апериодична, обладает хотя бъ одной малой функиией и имеет параметр сходимости $R=1$; б) принадлежит классу $\mathscr{X}$. Пусть выполняется условие (2A) с такими $f \in \mathscr{B}_{+} u \nu \in \mathscr{P}$, что $\xi(G f)>0 u \nu \ll \xi$, где $\xi \in \mathscr{M}_{0}$. Тогда для всех $\mu \in \mathscr{P}_{1}(\nu)$ имеем $\mu\left(Q^{n+1} f\right) \sim \mu\left(Q^{n} f\right)$, ито при $m=l+1$ (см. определение класса $\mathscr{X}$ ) равносильно (2.3).

Здесь требование $m=l+1$ можно заменить более общим предположением $\left(\mathrm{IV}^{\prime}\right)$ из [11]. Отметим также, что при выводе $\left(2 Б_{1}\right)$ может оказаться полезной лемма 5.2 из [11].

По поводу условия, включенного в первую фразу следующего предложения, см. теорему 4.1.

Предложение 4.3. Пусть имеет место (1.3) для всех $\nu \in \mathscr{P} u$ $f \in \mathscr{B}_{+} c \xi(G f)>0$, где $\xi \in \mathscr{M}_{0}$. Если $\nu$ u $f$ выббраны указанным образом и при этом справедливо соотношение (2.3) с $\mu=\nu$, то оно же выполняется и с любыми $\mu \in \mathscr{P}_{1}(\eta)$, для которых

$$
\varliminf_{n \rightarrow \infty} \frac{\mu\left(P^{n} f\right)}{\nu\left(P^{n} f\right)}>0 .
$$

Легко видеть, что (4.1) применимо, например, если $\mu \geqslant c \nu$ при каком-либо $c>0$ или если $\varliminf_{n \rightarrow \infty} f_{n}>0$ на некотором $A \subset E$ с $\mu(A)>0$, где $f_{n}$ определено так же, как в $\S 2$.

3. Приведем доказательства последних предложений.

Доказатель ство п редл о жения 4.1. Зададим $m \geqslant 0$ и $\varepsilon>0$, положим $\mu=\nu P^{m}$ и заметим, что $\mu=h \xi$, где $h \in \mathscr{B}_{+}$ (см. (3.4)) и $h=0$ вне $E_{i}$ при некотором $i \geqslant 1$. Пусть $\xi_{B}(A)=\xi(A \cap B)$ и $\mu_{B}(A)=\mu(A \cap B), A \in \mathscr{E}$, если множество $B$ измеримо. Равномерная интегрируемость семейства $F$ по $\xi_{E_{i}}$ позволяет найти $a>0$, при котором $a \xi_{E_{i}}\left(f_{n}\right)<\varepsilon, n \geqslant n_{0}$. При этом $\mu_{D} \leqslant a \xi_{D} \leqslant a \xi_{E_{i}}$, где $D=\left\{x \in E_{i}: h(x) \leqslant a\right\}$, и, следовательно, $\mu_{D}\left(f_{n}\right)<\varepsilon, n \geqslant n_{0}$. Неравенства же $a \xi_{F} \leqslant \mu_{F} \leqslant b \xi_{F}$, где $F=E_{i} \backslash D$ и $b=\sup h$, влекут равномерную интегрируемость $\mathscr{F}$ по $\mu_{F}$, и для завершения доказательства остается воспользоваться равенством $\mu=\mu_{D}+\mu_{F}$.

Доказательство предложения 4.2. Пусть $\varphi_{n}=$ $Q^{n} f / \nu\left(Q^{n} f\right), n \geqslant n_{0}$. Согласно лемме 4.1 из [11] (замечание к ее доказательству см. в $[12, \S 6])$ и теореме 4 из [13], отношения $Q^{n+1} f / Q^{n} f$ 
сходятся в $E$ к 1 при $n \rightarrow \infty$, и по теореме Егорова найдутся измеримые множества $E_{1} \subset E_{2} \subset \cdots$, на каждом из которых эта сходимость равномерна, тогда как $\eta\left(E \backslash E_{n}^{*}\right)^{-} \rightarrow 0$, а потому и $\mu\left(E \backslash E_{n}\right) \rightarrow 0$ для всех $\mu \in \mathscr{P}_{1}(\nu)$. По лемме 3.3 любая последовательность вида $\left\{\varphi_{n} ; n \in \mathfrak{N}\right\}$ содержит подпоследовательность $\left\{\varphi_{n} ; n \in \mathfrak{M}\right\}$, для которой $P^{m} \varphi_{n} \stackrel{\text { w }}{\longrightarrow} P^{m} F$ в $L(\mu), n \in \mathfrak{M}$, если $m \geqslant 0, \mu \in \mathscr{P}_{1}(\nu)$, а функция $F$ выбрана должным образом. Так как $\nu\left(\varphi_{n}\right)=1$ при $n \geqslant n_{0}$, отсюда следует равенство $\nu(F)=1$, в силу которого $\xi(F)>0$ (напомним, что $\nu \ll \xi)$. Тем самым с учетом апериодичности $X$ доказано, что $Q^{n} F(x)>0$ при всех $x \in E$ и $n$, превышающих некоторое натуральное $n(x)$.

Теперь, задав $\mu \in \mathscr{P}_{1}(\nu)$, подберем $m \geqslant 1$ из условия $\mu\left(Q^{m} F\right)>0$ (такое $m$ назовем допустимым). Тогда $\mu\left(1_{E_{k}} Q^{m} F\right)>0$ для всех достаточно больших $k$, и для них же

$$
\frac{\mu\left(1_{E_{k}} Q^{m-1} \varphi_{n}\right)}{\mu\left(1_{E_{k}} Q^{m} \varphi_{n}\right)} \rightarrow \frac{\mu\left(1_{E_{k}} Q^{m-1} F\right)}{\mu\left(1_{E_{k}} Q^{m} F\right)},
$$

если $n \in \mathfrak{M}$ стремится к $\infty$. Одновременно $Q^{m-1} \varphi_{n} / Q^{m} \varphi_{n}=$ $Q^{m+n-1} f / Q^{m+n} f$ стремится к 1 равномерно на $E_{k}$, так что левая часть (4.2) стремится к 1 , но поскольку правая часть не зависит от $n$, то эта часть обязана равняться 1 . Благодаря этому $\mu\left(1_{E_{k}} Q^{m-1} F\right)>0$ при достаточно больших $k$, т.е. вместе с $m$ допустимыми являются и все не превышающие его натуральные числа. Поэтому можно взять $m=1$ и, учитывая наше заключение относительно правой части (4.2), получить для достаточно больших $k$ равенство $\mu\left(1_{E_{k}} Q F\right)=\mu\left(1_{E_{k}} F\right)$, в силу которого $\mu(Q F)=\mu(F)$. Следовательно, $\mu\left(Q^{n+1} f\right) / \mu\left(Q^{n} f\right)=$ $\mu\left(Q \varphi_{n}\right) / \mu\left(\varphi_{n}\right) \rightarrow 1$, если $n \rightarrow \infty$ вдоль $\mathfrak{M}$. Имеющаяся свобода в выборе $\mathfrak{N}$ и $\mathfrak{M}$, как и при доказательстве теоремы 2.1 , влечет требуемое утверждение.

Д оказательст в о предл ож ения 4.3. Пусть, вопреки нашему утверждению, соотношение (2.3) нарушается для какой-либо меры $\mu \in \mathscr{P}_{1}(\eta)$, удовлетворяющей (4.1). Тогда $\mu\left(P^{n+1} f\right) / \mu\left(P^{n} f\right)>1+\delta$ при некотором $\delta>0$, если $n$ пробегает некоторую возрастающую последовательность $\mathfrak{N}$. Поскольку $\mu \in \mathscr{P}_{1}(\eta)$, найдутся такие $a, c>0$, что $\widetilde{\nu} \in \mathscr{P}_{\text {и }} \mu_{1} \leqslant \frac{1}{2} \widetilde{\nu}$, где $\widetilde{\nu}=a \eta$ и $\mu_{1}=c \mu$. Соотношения $(2.3)$ и (1.3) продолжают действовать, если $\nu$ в первом из них заменить на $\widetilde{\nu}$, а во втором - на $\nu_{1}=\widetilde{\nu}-\mu_{1} \geqslant \frac{1}{2} \widetilde{\nu}$. Поэтому существуют $a_{n} \geqslant 0$ и $b_{n} \geqslant 0$, $n \geqslant 1$, стремящиеся к 1 при $n \rightarrow \infty$ и такие, что

$$
\widetilde{\nu}\left(P^{n+1} f\right)=a_{n} \widetilde{\nu}\left(P^{n} f\right)=a_{n} \mu_{1}\left(P^{n} f\right)+a_{n} \nu_{1}\left(P^{n} f\right)
$$

и $\nu_{1}\left(P^{n} f\right) \geqslant b_{n} \nu_{1}\left(P^{n} f\right)$. Последнее неравенство показывает, что

$$
\widetilde{\nu}\left(P^{n+1} f\right)=\mu_{1}\left(P^{n+1} f\right)+\nu_{1}\left(P^{n+1} f\right) \geqslant(1+\delta) \mu_{1}\left(P^{n} f\right)+b_{n} \nu_{1}\left(P^{n} f\right),
$$


где $n \in \mathfrak{N}$. Сравнение (4.3) и (4.4) приводит к соотношениям

$$
\gamma_{n} \nu_{1}\left(P^{n} f\right) \geqslant\left(1+\delta-a_{n}\right) \mu_{1}\left(P^{n} f\right) \geqslant \frac{\delta}{2} \mu_{1}\left(P^{n} f\right)
$$

для достаточно больших $n \in \mathfrak{N}$, где $\gamma_{n}=a_{n}-b_{n}$. Таким образом, для тех же $n$ получаем неравенство $\gamma_{n} \widetilde{\nu}\left(P^{n} f\right) \geqslant \delta \mu\left(P^{n} f\right) / 2$, которое несовместимо с (4.1), поскольку $\gamma_{n} \rightarrow 0$ и, как легко проверить, $\widetilde{\nu}\left(P^{n} f\right) \sim \nu\left(P^{n} f\right)$. Тем самым доказательство завершено.

В заключение автор выражает благодарность рецензенту за полезные замечания.

\section{СПИСОК ЛИТЕРАТУРЫ}

1. Dellacherie C., Meyer P.-A. Probabilités et potentiel. Paris: Hermann, 1975, 291 p.

2. Guivarc' $h Y$. Théorèmes quotients pour les marches aléatoires. - Astérisque, 1980, v. 74 , p. $15-28$.

3. Lin M. Strong ratio limit theorems for mixing Markov operators. - Ann. Inst. H. Poincaré, 1976, v. 12, № 2, p. 181-191.

4. Orey S. Lecture Notes on Limit Theorems for Markov Chain Transition Probabilities. London: Van Nostrand, 1971, 108 p.

5. Данфорд Р., Швари Дж. Т. Линейные операторы. Общая теория. Т. 1, М.: ИЛ, $1962,895 \mathrm{c}$.

6. Молчанов C.A. Предельная теорема для отношений переходных вероятностей. Успехи матем. наук, 1967, т. 22, в. 2, с. 124-125.

7. Невё Ж. Математические основы теории вероятностей. М.: Мир, 1969, 309 с.

8. Нуммелин Э. Общие неприводимые цепи Маркова и неотрицательные операторы. М.: Мир, 1989, 207 с.

9. Ревюз Д. Цепи Маркова. М.: РФФИ, 1997, 431 с.

10. Чжун К.-Л. Однородные цепи Маркова. М.: Мир, 1964, 421 с.

11. Шур М.Г. Асимптотические свойства степеней положительных операторов. I, II. - Теория вероятн. и ее примен., 1984, т. 29, в. 4, с. $692-702$, 1985, т. 30 , в. 2 , с. $241-251$.

12. Шур М.Г. К предельным теоремам для отношений, порожденных случайными блужданиями в однородных пространствах. I, II. - Теория вероятн. и ее примен., 1988 , т. 33 , в. 4 , с. $706-719,1989$, т. 34 , в. 3 , с. $516-527$.

13. Шур М. Г. Об условии Лина в сильных предельных теоремах для отношений. Матем. заметки, 2004, т. 75 , в. 6 , с. 927-940.

14. Shur M. G. New ratio limit theorems for Markov chains. - Amer. Math. Soc. Transl. Ser. 2, 2002, v. 207, p. 203-212.

15. Woess W. Random walks on infinite graphs and groups - a survey on selected topics. - Bull. London Math. Soc., 1994, v. 26, № 1, p. 1-60.

16. Спичер Ф. Принципы случайного блуждания. М.: Мир, 1969, 472 с.

Поступила в редакцию 23.III.2004

Исправленный вариант 15.II. 2005 